\title{
Operating during COVID-19: Is there a risk of viral transmission from surgical smoke during surgery?
}

\author{
Phil Vourtzoumis, MDCM \\ Nawar Alkhamesi, MD, PhD \\ Ahmad Elnahas, MD, MSc \\ Jeffrey E. Hawel, MD \\ Christopher Schlachta, MDCM
}

Accepted May 11, 2020

\author{
Correspondence to: \\ P. Vourtzoumis \\ Department of General Surgery \\ Western University \\ 339 Windermere Rd. \\ London ON N6A 5A5 \\ pvourtzo@uwo.ca
}

DOI: $10.1503 /$ cjs. 007020

\section{SUMmaRY}

The World Health Organization declared a pandemic when coronavirus disease 2019 (COVID-19) started to sweep the globe. Growing concerns for the safety of health care workers was raised when up to $80 \%$ of people with COVID-19 showed mild or no symptoms at all. Some surgical procedures will be inevitable during the pandemic, and proper safety measures must be in place to avoid transmission risks. Surgical smoke is a common by-product from the use of energy devices in the operating room. The effects of surgical smoke have been studied for more than 40 years, and potential health hazards have been reported. Chemicals, carcinogens and biologically active materials, such as bacteria and viruses, have been isolated in surgical smoke. To ensure the safety of operating room personnel, we must consider whether there is any concern of viral transmission from the inhalation of surgical smoke.

\section{I}

n December 2019, an outbreak of coronavirus disease 2019 (COVID$19)$, caused by the novel severe acute respiratory syndrome coronavirus 2 (SARS-CoV-2), originated in Wuhan, China. On March 11, 2020, COVID-19 was declared a pandemic by the World Health Organization (WHO). As this pandemic sweeps the globe, concerns are being raised about the risk to health care workers treating patients who are known and unknown carriers of this highly contagious disease.

Initial estimates suggest that up to $80 \%$ of all patients with COVID-19 have either mild or no symptoms at all, and carriers are likely transmitting SARS-CoV-2 to others. The conventional routes via respiratory droplets and direct contact are the main mechanisms believed to be involved in transmission. The virus has also been detected in the gastrointestinal tract, blood, saliva and urine, broadening the potential routes of transmission. ${ }^{1}$ Reports by the Chinese Center for Disease Control and Prevention also mention the likelihood of aerosol transmission, which can occur during aerosol-generating medical procedures (AGMP). The surgical community is also faced with another potential route of virus transmission concern: surgical smoke.

Dissection or cauterization of tissue using heat-generating devices produce a gaseous by-product known as surgical smoke. In general, the heat causes rupture of the target cell membrane, generating a plume of smoke made up of $95 \%$ water and $5 \%$ cellular debris. About $77 \%$ of particulate matter inside smoke plume has a mean diameter of $0.07 \mu \mathrm{m}$, and particulate matter $<5 \mu \mathrm{m}$ is not filtered by surgical masks and may be inhaled by personnel in the operating room (OR). ${ }^{2}$

Many reviews in the past 40 years have looked at the potential harmful effects of prolonged exposure to surgical smoke. Approximately 500000 personnel, including surgeons, nurses, anesthesiologists and technicians, are exposed to surgical smoke in the OR each year in the United States. To understand whether there are any harmful effects associated with the 
inhalation of surgical smoke, many studies have isolated the smoke and examined its makeup. They found it can contain various chemicals, carcinogens, mutagens and biological components, such as malignant cells, bacteria and, more importantly for our focus, viruses. ${ }^{3}$ A concern amid this pandemic is whether viral transmission of SARS-CoV-2 from surgical smoke is possible.

Most of the previous literature revolves around the occupational risks of the human papillomavirus (HPV) to medical personnel. In 1988, Garden and colleagues ${ }^{4}$ found that intact viral DNA was present in surgical smoke and later showed that the reinoculation of laser smoke plume from bovine papillomavirus into calves produced papillomavirus-related tumours, therefore, suggesting a possible harmful transmission effect in humans. Most studies can prove that HPV is present in surgical smoke; however, the challenge faced by the medical community is whether this has any real clinical significance.

There have been 4 reported cases in the literature that suggest a likely occupational causality given significant exposure risk to HPV: a 44-year-old gynecologist who developed laryngeal papillomatosis, a 28-year-old gynecology OR nurse with recurrent and histologically proven laryngeal papillomatosis, a 53-year-old gynecologist who treated more than 3000 dysplastic cervical and vulvar lesions developed HPV-related tonsillar squamous cell carcinoma, and a 62-year-old gynecologist who developed HPV-related base of tongue cancer. ${ }^{2}$ Notably, these individuals did not have a significant history or any risk factors other than their long-term occupational exposure.

A recent study tested surgeons treating 134 patients who underwent cervical loop electrosurgical excision procedures (LEEP) for HPV. 5 The study assessed the surgical smoke, surgeon nasal swabs, the effect of smoke evacuation on HPV in plumes and the types of surgical masks used. The surgical smoke plume of 40 patients (30\%) was positive for HPV DNA. From these positive cases, 2 surgeons had appropriate HPV subtype detected on nasal swabs. Interestingly, the 2 surgeons who wore surgical masks had HPV detected, whereas those who wore N95 masks did not. Finally, the presence of HPV in smoke was inversely associated with the distance of the suction device from the surgical site.

In 1991, a pellet of cultured cells infected with human immunodeficiency viruse (HIV) was vapourized with a carbon dioxide laser. ${ }^{6}$ The smoke debris was collected using silastic tubing as a smoke evacuation device. There was no HIV DNA detected on culture medium flasks; however, there was HIV DNA present in 3 of 12 silastic tubes at 1 week and in 1 of 12 tubes at 2 weeks. Although this experiment demonstrated the presence of HIV in vapourized laser smoke from cell pellets, it is difficult to extrapolate given that no further studies, either in vitro or in vivo, have been performed.

Kwak and colleagues ${ }^{7}$ investigated whether hepatitis $B$ virus (HBV) could be detected in aerosol form. They isolated surgical smoke from 11 patients undergoing robotic or laparoscopic abdominal surgeries, and 10 of 11 patients had HBV DNA detected by polymerase chain reaction (PCR). Theirs was the first and only study to report $\mathrm{HBV}$ in smoke plumes, especially during laparoscopic surgery. There are no reports, however, of $\mathrm{HBV}$ transmission during open or laparoscopic surgery secondary to surgical smoke.

Several articles have described surgical smoke encountered during laparoscopic surgery. Similar to open surgery, the concern in laparoscopy is that the smoke is confined in the abdominal cavity and over time may be hazardous to the patient or OR personnel. No study to date has been able to demonstrate any long-term negative impact. Importantly, most studies suggested that proper laparoscopic smoke evacuation via filter, either passive or active, is adequate to ensure effective protection from potential hazardous surgical smoke. ${ }^{8}$

Operating room smoke, whether from open or laparoscopic procedures, should be evacuated in an effective and safe manner. The Association of periOperative Registered Nurses (AORN) group are known to advocate for a safe and smoke-free OR environment. ${ }^{9}$ They support the use of local exhaust ventilation, either by a central smoke evacuation system, portable smoke evacuation unit, wall suction with inline filter or laparoscopic evacuation/filtration system. For open surgery, the evacuation apparatus should be no more than 2 inches from the source of generated smoke. With regards to laparoscopy, automatic smoke evacuation not only decreases the risk of exposure to harmful chemicals, but also provides a better view of the operating field. It is also believed that without proper smoke evacuation measures, electrosurgical devices in procedures open to the OR can be more harmful than robotic or laparoscopic procedures with regard to exposure of uncontrolled surgical smoke emission. ${ }^{9}$

Despite all the literature suggesting that surgical smoke can have potential harmful effects with prolonged exposure, compliance with best practice measures has been difficult. The recommendations from various organizations advocating for local exhaust ventilators and respiratory precautions have been poorly implemented for many reasons, such as cost, lack of equipment or repair parts, physician resistance, staff complacency and extra personal accommodation devices. ${ }^{10}$

The COVID-19 pandemic has had a substantial impact on the current standards for patient care and management. To ensure the safety of patients and 
health care workers, we must quickly adapt and standardize practice as a surgical community. Given the novelty of the virus, the goal of this article was to address any concerns of whether transmission of SARS$\mathrm{CoV}-2$ from surgical smoke is possible. Inhalation of surgical smoke over a long period of time has been suspected to be hazardous to OR personnel. Most of the research conducted in the past 40 years mentions that more robust epidemiological studies need to be performed to attempt to demonstrate any true association of harm.

Focusing on the viral contents of surgical smoke, most of the literature to date has focused on exposure in gynecologists. As mentioned previously, there have been 4 documented cases of health care workers with prolonged exposure to surgical smoke who have developed HPV-related lesions or cancers. The common risk factor of prolonged exposure in these 4 individuals allows us to infer that surgical smoke exposure was the source of virus transmission. It was interesting to note through the various studies that wearing N95 masks rather than surgical masks and availability of effective smoke evacuation in the OR appeared to be protective factors. Other viruses found in surgical smoke were HIV and HBV. The studies on HIV used experimental in vitro models; therefore, the results were difficult to extrapolate to humans. With regards to $\mathrm{HBV}$, the virus was found in surgical smoke during laparoscopic surgery in patients known to be $\mathrm{HBV}$-positive. This raises the concern for possible transmission to OR personnel, but this has never been investigated.

There is minimal evidence to suggest that hazardous viral transmission is a common occupational health issue. There does not seem to be an epidemiological problem, but that does not mean it's impossible. Given the number of operative procedures worldwide that involve energy devices, it is plausible that more than 4 cases of questionable transmission could be identified.

Can the association between surgical smoke and virus transmission from case reports be extrapolated to suggest that SARS-CoV-2 may be transmitted to surgical personnel from surgical smoke? The conditions from previous studies and case reports differ from our current situation based on the type of virus, route of transmission, contagiousness of the virus, ability to withstand a given energy device and the period of exposure. Therefore, based on the available research it is quite difficult to interpret the data in relation to the COVID-19 pandemic. More importantly, there have been no documented cases of viral transmission from surgical smoke in previous pandemic experiences.

\section{Conclusion}

Information with respect to the COVID-19 pandemic is constantly evolving as we continue to work through this difficult experience. It is important to be aware of surgical smoke in the OR and the potential hazard associated with its presence. The risk of viral transmission via surgical smoke is unclear; however, surgeons and OR personnel should minimize any chance of surgical smoke transmission by employing safe personal protective and respiratory measures when performing open and minimally invasive surgery. This pandemic experience should highlight to the surgical community that further research and expansion of our knowledge on surgical smoke is necessary to build more robust safety guidelines moving forward.

Affiliations: From the Department of General Surgery, Schulich School of Medicine and Dentistry, Western University, London, Ont.

Competing interests: None declared.

Contributors: All authors contributed substantially to the conception, writing and revision of this article and approved the final version for publication.

\section{References}

1. Pan L, Mu M, Yang P, et al. Clinical characteristics of COVID-19 patients with digestive symptoms in Hubei, China: a descriptive, crosssectional, multicenter study. Am 7 Gastroenterol 2020;115:766-773.

2. Liu Y, Song Y, Hu X, et al. Awareness of surgical smoke hazards and enhancement of surgical smoke prevention among the gynecologists. 7 Cancer 2019;10:2788-99.

3. Limchantra IV, Fong Y, Melstrom KA. Surgical smoke exposure in operating room personnel: a review. FAMA Surg 2019; doi:10.1001/ jamasurg.2019.251.

4. Garden JM, O'Banion MK, Bakus $\mathrm{AD}$, et al. Viral disease transmitted by laser-generated plume (aerosol). Arch Dermatol 2002; 138:1303-7.

5. Zhou Q, Hu X, Zhou J, et al. Human papillomavirus DNA in surgical smoke during cervical loop electrosurgical excision procedures and its impact on the surgeon. Cancer Manag Res 2019;11:3643-54.

6. Baggish MS, Poiesz BJ, Joret D, et al. Presence of human immunodeficiency virus DNA in laser smoke. Lasers Surg Med 1991;11:197-203.

7. Kwak HD, Kim S-H, Seo YS, et al. Detecting hepatitis B virus in surgical smoke emitted during laparoscopic surgery. Occup Environ Med 2016;73:857-63.

8. Dobrogowski M, Wesolowski W, Kucharska M, et al. Chemical composition of surgical smoke formed in the abdominal cavity during laparoscopic cholecystectomy-assessment of the risk to the patient. Int 7 Occup Med Environ Health 2014;27:314-25.

9. Barnes S, Twomey C, Carrico R, et al. OR air quality: Is it time to consider adjunctive air cleaning technology? AORN F 2018;108:503-15.

10. Ilce A, Yuzden GE, Yavuz van Giersbergen M. The examination of problems experienced by nurses and doctors associated with exposure to surgical smoke and the necessary precautions. 7 Clin Nurs 2017;26:1555-61. 